\title{
The prevalence and incidence of musculoskeletal symptoms among hospital physicians: a systematic review
}

\author{
Karen M. Oude Hengel · Bart Visser • \\ Judith K. Sluiter
}

Received: 8 January 2010/Accepted: 21 July 2010/Published online: 5 August 2010

(C) The Author(s) 2010. This article is published with open access at Springerlink.com

\begin{abstract}
Objective Physicians are exposed to a range of workrelated risk factors that may result in occupational diseases. This systematic review aims at shedding light on the prevalence and incidence of musculoskeletal complaints among hospital physicians.

Methods A systematic literature search was performed in Pubmed and EMBASE (1990-2010), and methodological quality criteria were applied. A search was done for musculoskeletal complaints.

Results Five medium-quality studies and three highquality studies were included in this review. The definitions and assessment used in the studies for musculoskeletal complaints were different. In short, the frequently reported prevalence for hand and wrist pain was $8-33$ and $0 \%, 17 \%$ for shoulder pain, and 9-28\% for neck pain. Moreover, the annual prevalence of low back pain was between 33 and $68 \%$.

Interpretation The limited number of studies makes it difficult to draw conclusions, and the results should be intepreted with care. In conclusion, musculoskeletal complaints may be work-related complaints in hospital physicians, which need future attention.
\end{abstract}

K. M. Oude Hengel · B. Visser

Research Institute MOVE, Faculty of Human Movement

Sciences, VU University, Amsterdam, The Netherlands

\section{J. K. Sluiter ( $\square)$}

Coronel Institute of Occupational Health, Academic Medical

Center, University of Amsterdam, PO Box 22700,

1100 DE Amsterdam, The Netherlands

e-mail: j.sluiter@amc.uva.nl
Keywords Hospital · Physicians · Musculoskeletal complaints

\section{Introduction}

Work-related complaints have become a major concern for employees, employers and governments because of their negative impact on the health and productivity of the employees (Fulton-Kehoe et al. 2000). In 2005, a total of $23 \%$ of EU27 workers reported work-related muscular pains in shoulders, neck and/or upper/lower limbs (EFILWC 2007). For nonfatal occupational injuries in the United States, $18.6 \%$ of all new cases occurred in the health care and social assistance sectors; hospitals even topped the list of nonfatal injuries and illnesses per year (US labour statistics 2005). Moreover, for the health care and social work professions, $50 \%$ of the absences due to sickness are caused at work or by work (European Communities 2004).

Health care workers are exposed to several factors that can explain the heightened risk for illnesses and sick leave. For example, the awkward work postures and manual material handling of hospital staff lead to an increased risk for occupational musculoskeletal injuries (e.g., Waters et al. 2007).

Currently, it is difficult to provide adequate prevention of work-related diseases in physicians because most reviews reporting on diseases and disorders are based on all health care workers and do not differentiate for physicians (Joshi et al. 2006; Bousquet et al. 2006). Physicians are exposed to factors at the workplace that may cause a broad range of psychological, biological, physical and chemical disorders and diseases.

One risk among hospital physicians is due to multiple physical exposures, e.g. in the operation room because of 
new working techniques like laparoscopy (Stomberg et al. 2010) or duration of keeping awkward postures or because of walking distance during work (Conzett-Baumann et al. 2009). These factors may lead to different complaints of the musculoskeletal system that are known to be related to hospital work. For example, complaints of the musculoskeletal system can occur in the upper extremities among surgeons as a result of precision work in an awkward position (Berguer et al. 1999). In time, this may lead to musculoskeletal disorders.

An overview of the incidence and the prevalence of musculoskeletal complaints among hospital physicians may lead to more adequate prevention of work-related diseases and consequently provide a safer and healthier environment for the physicians. This systematic review aims at gaining insight into the prevalence and incidence of musculoskeletal complaints among hospital physicians.

\section{Methods}

\section{Search strategy}

The literature search included a computerized database search and a reference search. The computerized literature search was conducted in Pubmed and EMBASE. The search strategy aimed at identifying all available published studies that reported data on the incidence and prevalence of work-related complaints among hospital physicians. The key words for the search were the following: (1) physicians and accompanying specialism (e.g., dermatologist, internist, physiatrist and oncologist), (2) musculoskeletal, (3) prevalence, incidence, and (4) medical center, hospital. To avoid many studies about patients, the key word patient was used in the search as a limitation. Subsequently, the reference results were examined for additional studies. One reviewer $(\mathrm{KOH})$ screened the obtained titles and abstracts for eligibility. Studies were eligible when all the inclusion criteria were met. When inclusion or exclusion could not be made on the title or abstract, the full article was retrieved to decide of the article was eligible for the review.

\section{Inclusion criteria}

Given the large number of papers, the first reviewer $(\mathrm{KOH})$ narrowed the selection of the papers used by applying the following inclusion criteria:

- musculoskeletal complaints were defined as musculoskeletal complaints, musculoskeletal symptoms or musculoskeletal disorders.

- the study should be published in English.

- the study was published between January 1990 and January 2010.
Methodological quality assessment

All the articles were selected on the basis of six inclusion criteria: (1) positive when a description and clarification was given for a disorder or disease; (2) description of the setting and location of the hospital (e.g. city of the hospital, type of hospital, size of hospital); (3) description of the physicians; (4) and (5) description of the instruments and statistics; (6) positive when the response rate was at least $50 \%$. These criteria were selected as important to make a proper comparison between the papers and the population. Studies were classified as high (5-6 criteria), medium (3-4 criteria) or low quality (1-2 criteria). Low-quality studies were excluded from this systematic review.

\section{Results}

Study selection

The computer-generated search resulted in 160 references in Pubmed and 157 references in EMBASE. After exclusion of the duplicated references, all titles and abstracts were checked for inclusion or exclusion. The most important reasons for exclusion were: (1) the study did not distinguish between health care workers in their study and (2) the study was reporting the prevalence of musculoskeletal disorders among patients instead of physicians. After selection based on the title and on the abstract, 13 articles were selected. After reviewing the whole paper, a total of five articles were included. Screening the reference list of the included articles provided an extra three studies. Finally, a total of eight studies were selected for this review (Fig. 1).

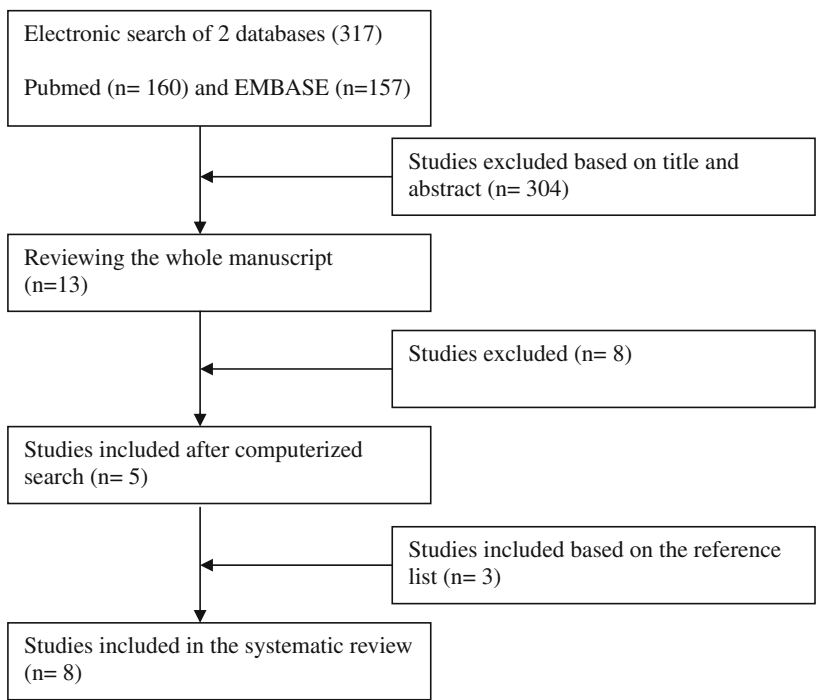

Fig. 1 Flowchart of selection process 
Methodological quality assessment

Table 1 showed the methodological quality assessment of the eight studies (Berguer et al. 1999; Cunningham et al. 2006; Failde et al. 2000; Johnston et al. 2005; Karahan et al. 2009; Smith et al. 2006; Szeto et al. 2009; Wolf et al. 2000). Five medium-quality studies (Berguer et al. 1999; Failde et al. 2000; Johnston et al. 2005; Karahan et al. 2009; Wolf et al. 2000) and three high-quality studies (Cunningham et al. 2006; Smith et al. 2006; Szeto et al. 2009) about the prevalence of musculoskeletal complaints in the upper extremities or in the back were included (Table 1). No studies were found on the incidence of musculoskeletal disorders among hospital physicians.

\section{Study characteristics}

Four studies reported musculoskeletal complaints among surgeons, three studies reported musculoskeletal complaints among all doctors and one study reported musculoskeletal complaints among urologists (Table 2). It should be noted that Johnston et al. (2005) reported an effect of two subgroups according to tasks performed in the operating room, hand-assisted laparoscopy and standard laparoscopy. The number of participants varied from 18 to 286 . The studies have been conducted in the United States of America (Berguer et al. 1999; Johnston et al. 2005; Wolf et al. 2000), Ireland (Cunningham et al. 2006), Spain (Failde et al. 2000), Turkey (Karahan et al. 2009) and China (Smith et al. 2006; Szeto et al. 2009).

Different definitions were used in the studies for musculoskeletal complaints. Cunningham et al. (2006) used the most broad definition of musculoskeletal complaints as they defined complaints as ache, pain or discomfort. Three other studies (Karahan et al. 2009; Smith et al. 2006; Szeto et al. 2009) defined musculoskeletal complaints as discomfort in the investigated body regions, whereas one study defined musculoskeletal complaints in term of pain

Table 1 Methodological criteria

\begin{tabular}{lllllllll}
\hline & Quality criteria & & & \\
\cline { 2 - 7 } & 1 & 2 & 3 & 4 & 5 & 6 & Score & Quality label \\
\hline Berguer (1999) & + & - & + & + & + & - & 4 & MQ \\
Cunningham (2006) & + & - & + & + & + & + & 5 & HQ \\
Failde (2000) & + & + & - & - & + & - & 3 & MQ \\
Johnston (2005) & + & - & + & - & + & + & 4 & MQ \\
Karahan (2009) & + & - & - & + & + & + & 4 & MQ \\
Smith (2006) & + & - & + & + & + & + & 5 & HQ \\
Szeto (2009) & + & + & + & + & + & - & 5 & HQ \\
Wolf (2000) & + & + & + & - & - & - & 3 & MQ \\
\hline
\end{tabular}

$H Q$ high quality, $M Q$ medium quality
(Berguer et al. 1999). Pain or muscular injury was used as a definition in another study (Johnston et al. 2005). Two studies reported no clear definition of musculoskeletal complaints (Failde et al. 2000; Wolf et al. 2000). Different types of prevalences have been assessed: point prevalence, annual prevalence and lifetime prevalence.

Besides different definitions used, musculoskeletal complaints were also assessed in different ways. Three studies used existing questionnaires: two of these studies used the Standardized Nordic Questionnaire (Smith et al. 2006; Szeto et al. 2009) and one study used the health and back pain survey (Cunningham et al. 2006). A self-formulated questionnaire was used in three studies (Berguer et al. 1999; Johnston et al. 2005; Karahan et al. 2009), whereas two studies (Failde et al. 2000; Wolf et al. 2000) did not report about the questions used.

Prevalence of musculoskeletal complaints

First, three medium-quality studies reported about the prevalence of hand and wrist pain. The results for the frequently reported prevalence of hand and wrist pain were found between 8 (Berguer et al. 1999) and 33\% (Johnston et al. 2005), and the occasionally reported prevalence of hand and wrist pain were 36 (Berguer et al. 1999) and 67\% (Wolf et al. 2000). Only Johnston et al. (2005) examined the frequently reported prevalence for forearm pain (25 and $4 \%$ ). Wolf et al. (2000) found an occasionally reported prevalence of elbow pain of $11 \%$.

Next, two medium-quality studies and two high-quality studies reported shoulder pain. Two studies found a frequently reported prevalence between 0 (Johnston et al. 2005) and 17\% (Wolf et al. 2000). Two studies reported about the annual prevalence for shoulder pain of 38 (Smith et al. 2006) and 58\% (Szeto et al. 2009). The occasionally and frequently reported prevalence of shoulder/arm pain was 43 and 12\%, respectively (Berguer et al. 1999).

Furthermore, neck pain was described by four studies (two medium-quality studies and two high-quality studies). They found frequently reported prevalences of 9 and $28 \%$ and an occasionally reported prevalence of $43 \%$. The annual prevalences of neck pain were 42 and $83 \%$.

Lastly, two medium-quality studies and three highquality studies reported a prevalence for back pain. Failde et al. (2000) found a prevalence for low back pain (LBP) of $80 \%$. Cunningham et al. (2006) reported a point prevalence for LBP of $24 \%$, an annual prevalence of $33 \%$ and a lifetime prevalence of $67 \%$. Compared to the annual prevalence for LBP of Cunningham, three other studies showed prevalences between 44 and 68\% (Karahan et al. 2009; Smith et al. 2006; Szeto et al. 2009). Wolf et al. (2000) reported an occasional prevalence of LBP of $33 \%$. Next to LBP, Smith et al. (2006) and Szeto et al. (2009) also 
Table 2 Eight studies that assessed frequently reported prevalence of musculoskeletal complaints. The study parameters of study design, sample size, type of doctors, country and prevalence are presented

\begin{tabular}{|c|c|c|c|c|c|c|c|c|c|c|c|}
\hline \multirow[t]{2}{*}{ First author } & \multirow[t]{2}{*}{$N$} & \multirow[t]{2}{*}{ Type } & \multirow[t]{2}{*}{ Country } & \multirow[t]{2}{*}{ Prevalence } & \multicolumn{7}{|l|}{$(\%)$} \\
\hline & & & & & $\begin{array}{l}\text { Hand/ } \\
\text { wrist }\end{array}$ & $\begin{array}{l}\text { Forearm/ } \\
\text { elbow }\end{array}$ & Shoulder & $\begin{array}{l}\text { Shoulder/ } \\
\text { arm }\end{array}$ & Neck & $\begin{array}{l}\text { Upper } \\
\text { back }\end{array}$ & LBP \\
\hline \multirow[t]{2}{*}{ Berguer (1999) } & 149 & Surgeons & USA & Occasional & 36 & & & 43 & 43 & & \\
\hline & & & & Frequent & 11 & & & 12 & 9 & & \\
\hline \multirow[t]{3}{*}{ Cunningham (2006) } & 21 & Physicians & Ireland & Point & & & & & & & 24 \\
\hline & & & & Annual & & & & & & & 33 \\
\hline & & & & Lifetime & & & & & & & 67 \\
\hline Failde (2000) & 94 & Physicians & Spain & NA & & & & & & & 80 \\
\hline \multirow[t]{2}{*}{ Johnston (2005) } & 25 (HAL) & Surgeons & USA & Frequent & 33 & 25 & 10 & & & & \\
\hline & $25(\mathrm{SL})$ & Surgeons & & Frequent & 8 & 4 & 0 & & & & \\
\hline Karahan (2009) & 90 & Physicians & Turkey & Annual & & & & & & & 63 \\
\hline Smith (2006) & 286 & Physicians & China & Annual & & & 38 & & 42 & 29 & 44 \\
\hline Szeto (2009) & 135 & Surgeons & China & Annual & & & 58 & & 83 & 53 & 68 \\
\hline \multirow[t]{2}{*}{ Wolf (2000) } & 18 & Urologists & USA & Occasional & 67 & 11 & & & & & 33 \\
\hline & & & & Frequent & & & 17 & 28 & & & \\
\hline
\end{tabular}

$H A L$ hand-assisted laparoscopy, $S L$ standard laparoscopy, $N L$ the Netherlands, $L B P$ low back pain, $N A$ not applicable

presented the annual prevalences of the upper back and this was 29 and $53 \%$.

\section{Discussion}

This review focused on the incidence and prevalence of musculoskeletal complaints among hospital physicians.

The review resulted in a broad range of prevalence of musculoskeletal complaints among different body regions. This could possibly be explained by the differences in the usage of different definition and questionnaires to assess musculoskeletal complaints. To illustrate, the study of Berguer et al. (1999) reported musculoskeletal complaints as pain, whereas Szeto et al. (2009) defined musculoskeletal complaints as discomfort. The different definitions and questionnaires that were used in both studies might be an explanation for the findings. Only three of the eight studies used existing questionnaires. Future research should focus on using validated questionnaires.

Musculoskeletal complaints seemed high. However, no comparison with the working population could be made because the case definitions of data from the general population were not assessed in similar ways over the different countries where the studies were executed. Clearly defined timeline was used in most of the studies included.

The information that was found in this review may form part of a base of knowledge in the specific groups of doctors examined, which is needed to prevent participation problems of medical doctors. Such a knowledge base should be based on valid assessment techniques and be useful in creating effective measures to: (1) keep workers healthy in their jobs; (2) increase the safety of (co)workers; and (3) optimize the person-job interaction (Sluiter and Frings-Dresen 2007; Sluiter 2006). Workers' Health Surveillance should be performed with the following purposes in mind for employees: (1) to identify individuals on a regular basis who may have developed a susceptibility to a known hazard in the workplace; (2) to screen out workers whose present health hinders them from performing their job as safely as other employees, thereby endangering themselves or others; or (3) to screen out those who are unlikely to perform satisfactorily due to a developed health problem (Sluiter and Frings-Dresen 2007).

It is important to note that the present review has limitations. First, some articles may have been missed by the chosen search strategy. Secondly, there were two factors that possibly lead to an underestimation of the prevalence or incidence of musculoskeletal complaints. First, studies only examining the physicians in their work setting and therefore sick-listed physicians were not included in the results. Second, junior doctors and residents who previously quit working due to their disorder or diseases were also not included in the results.

Because relatively few studies were found on the prevalence and no studies were found on incidence of workrelated musculoskeletal complaints among hospital physicians, more research over time is needed to have a more complete overview of all relevant musculoskeletal diseases and disorders. In addition, research should determine 
differences between medical specialties. Distinguishing between physicians could lead to a more specific overview and therefore to better prevention.

In conclusion, musculoskeletal complaints may be work-related complaints because of specific risk factors for hospital physicians, which need future attention as well.

Conflict of interest The authors declare that they have no conflict of interest.

Open Access This article is distributed under the terms of the Creative Commons Attribution Noncommercial License which permits any noncommercial use, distribution, and reproduction in any medium, provided the original author(s) and source are credited.

\section{References}

Berguer R, Forkey DL, Smith WD (1999) Ergonomic problems associated with laparoscopic surgery. Surg Endosc 13(5):466-468

Bousquet J, Flahault A, Vandenplas O, Ameille J, Duron JJ, Pecquet C, Chevrie K, Annesi-Maesano I (2006) Natural rubber latex allergy among health care workers: a systematic review of the evidence. J Allergy Clin Immunol 118:447-454

Conzett-Baumann K, Jaggi GP, Hüsler A, Hüsler J, Beer JH (2009) The daily walking distance of young doctors and their body mass index. Eur J Int Med 20(6):622-624

Cunningham C, Flynn T, Blake C (2006) Low back pain and occupation among Irish health service workers. Occup Med 56:447-454

EFILWC (2007) Fourth European working conditions survey. European Foundation for the Improvement of Living and Working Conditions, Dublin. ISBN 92-897-0974-X

European Communities (2004) Work and health in the EU, a statistical portrait. European Communities

Failde I, Gonzalez JL, Novalbos JP, Casais F, Marín J, Elorza J (2000) Psychological and occupational predictive factors for back pain among employees of a university hospital in southern Spain. Occup Med 50:591-596
Fulton-Kehoe D, Franklin G, Weaver M, Cheadle A (2000) Years of productivity lost among injured workers in Washington State: modeling disability burden in workers' compensation. Am J Ind Med 37:656-662

Johnston WK, Hollenbeck BK, Wolf JS (2005) Comparison of neuromuscular injuries to the surgeon during hand-assisted and standard laparoscopic urologic surgery. J Endourol 19(3): 377-381

Joshi R, Reingold AL, Menzies D, Pai M (2006) Tuberculosis among health care workers in low-and middle-income countries: a systematic review. PLoS Med 3:e494

Karahan A, Kav S, Abbasoglu A, Dogan N (2009) Low back pain: prevalence and associated risk factors among hospital staff. J Adv Nurs 65:516-524

Labour statistics (2005) Workplace injuries and illnesses in 2005. Department of labour, United States

Sluiter JK (2006) High-demand jobs: age-related diversity in work ability? Appl Ergon 37:429-440

Sluiter JK, Frings-Dresen MH (2007) What do we know about ageing at work? Evidence-based fitness for duty and health in fire fighters. Ergonomics 50:1897-1913

Smith DR, Wei N, Zhang YJ, Wang RS (2006) Musculoskeletal complaints and psychosocial risk factors among physicians in mainland China. Int J Indus Ergon 36:599-603

Stomberg MW, Tronstad SE, Hedberg K, Bengtsson J, Jonsson P, Johansen L, Lindvall B (2010) Work-related musculoskeletal disorders when performing laparoscopic surgery. Surg Laparosc Endosc Percutan Tech 20:49-53

Szeto GP, Ho P, Ting AC, Poon JT, Cheng SW, Tsang RC (2009) Work-related musculoskeletal symptoms in surgeons. J Occup Rehabil 19:175-184

Waters TR, Nelson A, Proctor C (2007) Patient handling tasks with high risk for musculoskeletal disorders in critical care. Crit Care Nurs Clin N Am 19:131-143

Wolf JS Jr, Marcovich R, Gill IS, Sung GT, Kavoussi LR, Clayman RV, McDougall EM, Shalhav A, Dunn MD, Afane JS, Moore RG, Parra RO, Winfield HN, Sosa RE, Chen RN, Moran ME, Nakada SY, Hamilton BD, Albala DM, Koleski F, Das S, Adams JB, Polascik TJ (2000) Survey of neuromuscular injuries to the patient and surgeon during urologic laparoscopic surgery. Urology 55:831-836 